\title{
Informational entropic Regge trajectories of meson families in AdS/QCD
}

\author{
A. E. Bernardini ${ }^{1, *}$ and R. da Rocha ${ }^{2, \dagger}$ \\ ${ }^{1}$ Departamento de Física, Universidade Federal de São Carlos, \\ P.O. Box 676, 13565-905 São Carlos, SP, Brazil \\ ${ }^{2}$ Federal University of $A B C$, Center of Mathematics, Computing and Cognition, \\ 09210-580 Santo André, Brazil
}

(Received 26 September 2018; published 20 December 2018)

\begin{abstract}
Bulk mesons propagating in chiral and gluon condensates, in a gravitational background, are scrutinized in holographic soft wall AdS/QCD models, involving deformed dilatonic backgrounds. The configurational entropy of the $a_{1}$ axial vector, the $\rho$ vector, and the $f_{0}$ scalar meson families is then computed. Two types of informational entropic Regge trajectories are then obtained, where the logarithm of the meson configurational entropy is expressed in terms of both the experimental meson mass spectra and their excitation number as well. Therefore, the mass spectra of the next generation of elements in each meson family, besides being predicted as eigenvalues of Schrödinger-like equations, are estimated with better accuracy and are discussed.
\end{abstract}

DOI: 10.1103/PhysRevD.98.126011

\section{INTRODUCTION}

The Shannon's information entropy paradigm resides in encoding information in stochastic processes [1]. The configurational entropy (CE) is a quantity that implements the information entropy as a measure that logarithmically evaluates the number of bits needed to designate the organization of a system. In particular, the CE comprehends the information compression into the configuration of wave modes in a physical system [2,3]. Among the socalled configurational information measures [4], the CE encompasses the informational quantification of the spatial complexity of a localized system [5,6]. The wave modes and particle excitations that are correlated to critical points of the $\mathrm{CE}$ have been shown to be more dominant or abundant among all other modes and, hence, more detectable or observable in nature [7-9]. The CE plays a relevant role in the study of phase transitions, which are also driven by critical points of the $\mathrm{CE}$, underlying diverse physical systems [5,6,10,11]. A meticulous overview of the information entropy formalism can be seen in Ref. [12].

Quantum chromodynamics (QCD) governs the strong interactions among gluons and quarks. The AdS/QCD

\footnotetext{
alexeb@ufscar.br

†roldao.rocha@ufabc.edu.br
}

Published by the American Physical Society under the terms of the Creative Commons Attribution 4.0 International license. Further distribution of this work must maintain attribution to the author(s) and the published article's title, journal citation, and DOI. Funded by SCOAP . holographic setup presents an $\mathrm{AdS}_{5}$ bulk, ${ }^{1}$ wherein gravity that is weakly coupled emulates the dual setup to the $4 \mathrm{~d}$ (conformal) field theory (CFT), which is strongly coupled on the $\mathrm{AdS}_{5}$ boundary. In the duality dictionary, physical fields in the $\mathrm{AdS}_{5}$ bulk are dual objects to $4 \mathrm{~d}$ operators of QCD [13]. It is worth mentioning that the bulk fifth dimension is nothing more than the energy scale of the theory. Confinement can then be implemented either by a Heaviside cutoff in the bulk - the hard wall $[14,15]$ —or by a dilatonic field, which accomplishes a smooth cutoff along the $\mathrm{AdS}_{5}$ bulk - the soft wall model [16,17]. From a phenomenological point of view, the quark-gluon plasma (QGP), the mesonic mass spectra and their Regge trajectories $[18,19]$, and other quantities were derived, using the holographic soft wall AdS/QCD, being precisely corroborated by experimental data [20]. Soft wall AdS/QCD models implement the (chiral) symmetry breaking [21,22] and confinement as well [17,23-25]. In particular, mesonic phenomenology can thus be allocated into the soft wall AdS/QCD. Throughout this paper, although QCD is driven by a $\mathrm{SU}(3)$ gauge symmetry, one can supersede it by a $\mathrm{SU}\left(N_{c}\right)$ group. Hence, the so-called 't Hooft large- $N_{c}$ limit [26] plays an important role on the soft wall.

In the context discussed above, the $\mathrm{CE}$ has been recently promoted to a relevant setup in the context of holographic AdS/QCD models. Besides probing important informational aspects of the AdS/QCD, the CE also supports some foundations to better understand mesonic states in QCD phenomenology. Some of the existing experimental data

\footnotetext{
${ }^{1}$ Anti-de Sitter.
} 
regarding meson families and glueball states were corroborated by the $\mathrm{CE}$, which points into the direction of the most abundant and dominant physical states in QCD, in an intense research program. In fact, holographic AdS/QCD models were first scrutinized, in the CE framework, in Refs. [7-9]. This subject was recently extended to baryons and exotic states in Ref. [27]. Mesonic excitations with lower $s$-wave resonances were proved, in Ref. [7], to present dominance over their higher $s$-wave counterparts. Thereafter, the CE paradigm was employed to scrutinize scalar glueball states in Ref. [8], corroborating with lattice and experimental data [20]. In addition, the CE of dynamical AdS/QCD with tachyonic potentials improved our understanding about meson phenomenology in Ref. [28]. Moreover, the $\mathrm{CE}$ that underlies bottomonium and charmonium states at zero temperature supports the experimental rareness of quarkonia states with higher masses, as discussed in Ref. [9]. The finite temperature case was then implemented in Ref. [29], with unexpected and relevant new features of quarkonia. Finally, QGPs with topological defects were also explored in the CE setup [30]. The CE also improved the understanding of the AdS/QCD lightfront wave function, where the color-glass condensate regime was used to study mesons in Refs. [31-34]. With the CE tools, heavy ion collisions were also studied [35]. Furthermore, aspects of the $\mathrm{CE}$ unraveled prominent features of the gravity side of AdS/QCD and related phenomena. The Hawking-Page phase transition was studied in Ref. [11] in the context of the CE. Posterior to the influential works [36,37], graviton condensates were explored by the CE, in the AdS/CFT membrane paradigm [38]. The CE apparatus was further employed in field theory, in various contexts [39-42]. Here we aim to take one big step further, besides corroborating the experimental data of the $a_{1}$ axial vector, the $\rho$ vector, and the $f_{0}$ scalar meson families. Using both the quadratic and deformed dilatonic backgrounds, informational entropic Regge trajectories are derived, relating the logarithm of the CE to the $n$ excitation number of mesonic states, for each one of the meson families. The meson mass spectra are well known to be predicted in the soft wall AdS/QCD, with good accuracy. In fact, for both the dilatonic backgrounds, the equations of motion (EOMs) of a graviton-dilaton-gluon action are equivalent to Schrödinger-like equations, whose eigenvalues consist of the meson squared mass spectra. Their eigenfunctions represent the mesonic states and excitations, for the $a_{1}$ axial vector, the $\rho$ vector, and the $f_{0}$ scalar meson families. The $\mathrm{CE}$ for these meson families are also computed with respect to the meson family mass spectra, revealing a second type of informational entropic Regge trajectory. Hence, one can extrapolate the meson mass spectra from these informational entropic Regge trajectories, also predicting the mass of the next generation of elements in each meson family, corresponding to higher $n$ excitation numbers, with good accuracy. The masses of the first mesonic excitations of the next generation, in each meson family, are then estimated and discussed. This article is organized as follows: Section II is devoted to briefly reviewing the soft wall AdS/QCD framework, mainly emphasizing the standard quadratic dilaton model. The mass spectra of the $\rho$ vector, the $a_{1}$ axial vector, and the $f_{0}$ scalar mesonic states are revised. Section III is dedicated to introducing the chiral and gluon condensates, with a two flavor system in the graviton-dilaton-gluon setup, for both the quadratic and deformed dilatonic fields. In Sec. IV, the $\mathrm{CE}$ is computed for the $a_{1}$ axial vector, the $\rho$ vector, and the $f_{0}$ scalar meson families, as a function of the $n$ excitation number. Hence, informational entropic Regge trajectories are read off from these calculations, showing a relation between the logarithm of the $\mathrm{CE}$, for all regarded meson families, and their $n$ excitation modes. In addition, the meson mass spectra for higher excitation numbers can also be extracted from a second kind of informational entropic Regge trajectory, which relates the logarithm of the CE and the meson mass spectra, for each meson family. Hence, the mass spectra of higher $n$ excited mesonic states, in each meson family, are estimated with good accuracy. In Sec. V, our concluding remarks, outlook, and perspectives are drawn.

\section{SOFT WALL ADS/QCD}

The $\mathrm{AdS}_{5}$ vacuum bulk has a $4 \mathrm{~d}$ boundary that supports gauge theory; it is conformally invariant, emulating the standard QCD when $N_{c} \gg 1$. The boundary conformal symmetry can be broken, making QCD describe the confinement. In this regime, gravity in the bulk is dual to the QCD at the boundary. A straightforward way to break the boundary symmetry is, for example, to endow the AdS bulk with a dilatonic field. Since QCD approximately recovers conformal symmetries in a high energy regime, then the pure AdS bulk must prevail in the ultraviolet (UV) regime. Meson families can be emulated in the holographic soft wall AdS/QCD [17,43] and in its extended versions $[21,24,44,45]$, including the dynamical models [28,46-49] and scalars and vector mesonic states [50]. The Regge trajectories for excited light-flavor mesons were originally derived in Ref. [17], on a soft wall model endowed with a quadratic dilaton, $\Phi(z)=\mu^{2} z^{2}$, where $\mu$ introduces an energy scale in QCD [51]. The $\mathrm{AdS}_{5}$ background bulk metric is expressed, in conformal coordinates, as

$$
d s^{2}=g_{m n} d x^{m} d x^{n}=e^{2 \mathrm{~A}(z)}\left(\eta_{\mu \nu} d x^{\mu} d x^{\nu}+d z^{2}\right),
$$

for the warp factor $\mathrm{A}(z)=-\log (z / \ell)$, where $\eta_{\mu \nu}$ denotes the $4 \mathrm{~d}$ space-time metric components and $\ell$ is related to the bulk curvature radius. Other warp factors, extending the soft wall AdS/QCD, were used in Refs. [47,48,52]. From here on, $m, n, q$ denote bulk indices, running from 0 to 4 , where $x^{m}=\left(x^{\mu}, x^{4}\right)$, for $x^{\mu}$ denoting $4 \mathrm{~d}$ space-time 
coordinates and $x^{4}$ denoting the bulk coordinate. Lightflavor mesonic excitations are represented by bulk $\mathfrak{X}(z)$ fields, which are objects dual to the quark-antiquark operator, with associated mass $m_{\mathfrak{X}}$, and governed by the action [17]

$$
S=-\int e^{-\Phi(z)} \sqrt{-g} \operatorname{Tr} \mathfrak{Q} d^{5} x
$$

where

$$
\mathfrak{Q}=D_{m} \mathfrak{X} D^{m} \mathfrak{X}+m_{\mathfrak{X}}^{2} \mathfrak{X}_{m} \mathfrak{X}^{m}+\frac{N_{c}}{48 \pi^{2}}\left(F_{R}^{2}+F_{L}^{2}\right),
$$

where the $A_{L}^{m}$ and $A_{R}^{m}$ gauge fields drive the $\mathrm{SU}(2)_{L} \times$ $\mathrm{SU}(2)_{R}$ chiral flavor symmetry of QCD. Each $\mathrm{SU}(2)$, with $\left\{f_{b} / 2\right\}(b=1,2,3)$ generators, corresponds to one quark flavor. The left and right gauge field strengths, respectively, read

$$
F_{L, R}^{m n}=\partial^{[m} A_{L, R}^{n]}-i\left[A_{L, R}^{m}, A_{L, R}^{n}\right],
$$

where $A_{L, R}^{m}=A_{L, R}^{m a} f_{a}$. The covariant derivative is explicitly given by $D^{m} \mathfrak{X}=\partial^{m} \mathfrak{X}-i A_{L}^{m} \mathfrak{X}+i \mathfrak{X} A_{R}^{m}$. The $\mathfrak{X}(z)$ field incorporates the $S$ (scalar) and $P$ (pseudoscalar) fields as [19]

$$
\mathfrak{X}(z)=(S+\xi(z)) \exp \left(i P^{b} t^{b}\right),
$$

where $\xi(z)$ is a vacuum expectation value that breaks chiral symmetry [19]. To describe the vector and axial vector mesons, the left, $A_{L}$, and right, $A_{R}$, gauge fields can be split into the vector $(V)$ and axial vector $(\AA)$ fields as [19]

$$
\begin{aligned}
& V^{m}=\left(A_{R}^{m}+A_{L}^{m}\right) / 2, \\
& \stackrel{\circ}{A}^{m}=\left(A_{R}^{m}-A_{L}^{m}\right) / 2,
\end{aligned}
$$

yielding the respective gauge field strengths,

$$
\begin{aligned}
& F_{V}^{m n}=\partial^{[m} V^{n]}-i\left[V^{m}, V^{n}\right], \\
& F_{\AA}^{m n}=\partial^{[m} \stackrel{\circ}{A}^{n]}-i\left[\stackrel{\circ}{A}^{m}, \stackrel{\circ}{ }^{n}\right] .
\end{aligned}
$$

With respect to the vector $V$ and axial vector $\stackrel{\circ}{A}$ fields, the soft wall Lagrangian (3) reads

$$
\mathfrak{L}=\mathrm{D}_{m} \mathfrak{X} \mathrm{D}^{m} \mathfrak{X}+m_{\mathfrak{X}}^{2} \mathfrak{X}_{m} \mathfrak{X}^{m}+\frac{N_{c}}{24 \pi^{2}}\left(F_{\AA}^{2}+F_{V}^{2}\right),
$$

for $\mathrm{D}_{m}=\partial_{m} \mathfrak{X}+i\left(X V_{m}-V_{m} X-A_{m} X-X A_{m}\right)$. The EOM for the $\xi(z)$ field then reads $\xi^{\prime \prime}(z)+\left(3 \mathrm{~A}^{\prime}(z)-\Phi^{\prime}(z)\right) \xi^{\prime}(z)-m_{\mathfrak{X}}^{2}(z) e^{2 \mathrm{~A}(z)} \xi(z)=0$.

In the standard soft wall model, $\Phi(z)=\mu^{2} z^{2}$ [17], and Eq. (10) has the solutions

$\xi(z)=c_{1} z^{3} \exp \left(z^{2} / 2\right) I(z)+c_{2} \mathrm{G}_{12}^{20}\left(\begin{array}{l|c}-z^{2} & 1 \\ 1 / 2,3 / 2\end{array}\right)$,

with $I(z)=I_{0}\left(\frac{z^{2}}{2}\right)+I_{1}\left(\frac{z^{2}}{2}\right)$, where $I_{n}(z)$ denotes the first kind of modified Bessel function, and the second term is the Meijer function of $\{1,2 ; 2,0\}$ order. We denote by $\mathrm{v}_{n}$ the functions that describe the $\rho$ vector mesons, and by $\mathrm{a}_{n}$ those that represent the $a_{1}$ axial vector mesons, whereas the $s_{n}$ denotes the functions that comprise the $f_{0}$ scalar mesons. For the vector $(\rho)$, axial vector $\left(a_{1}\right)$, and scalar $\left(f_{0}\right)$ meson families, for $n=1,2, \ldots$, the meson spectra in the standard soft wall AdS/QCD model are ruled by the following EOMs ${ }^{2}$ :

$$
\begin{aligned}
& \left(-\frac{d^{2}}{d z^{2}}+V_{\mathrm{s}}(z)\right) \mathrm{s}_{n}(z)=m_{n}^{2} \mathrm{~s}_{n}(z), \\
& \left(-\frac{d^{2}}{d z^{2}}+V_{\rho}(z)\right) \mathrm{v}_{n}(z)=m_{n}^{2} \mathrm{v}_{n}(z), \\
& \left(-\frac{d^{2}}{d z^{2}}+V_{\mathrm{a}}(z)\right) \mathrm{a}_{n}(z)=m_{n}^{2} \mathrm{a}_{n}(z),
\end{aligned}
$$

where the Schrödinger-like potentials are, respectively, given by

\footnotetext{
${ }^{2}$ At this point, a clarifying note relative to Eq. (14) from Ref. [17] is necessary. For mode functions $\mathrm{V}_{n}(z)$ identified by the quantum mechanical wave functions, $\psi_{n}(z)$, i.e., $\mathrm{v}_{n}(z)=$ $\exp \left(z^{2} / 2\right) z^{1 / 2} \psi_{n}(z)$, in the literature, one frequently notices that $n=1$ refers to the ground state of the quantum system (to be a clear reference to the hydrogen atom quantum mechanics, a standard notation in the literature). Therefore, the right expression
} for $\psi_{n}(z)$ (a corrected one from Ref. [17]) should be given by

$$
\psi_{n}(z)=e^{-z^{2} / 2} z^{m+1 / 2} \sqrt{\frac{2(n-1) !}{(m+n-1) !}} L_{n-1}^{m}\left(z^{2}\right),
$$

such that $I_{\psi} \equiv \int_{0}^{\infty} d z \psi_{n}^{*}(z) \psi_{n}(z)$ reads

$$
\begin{aligned}
I_{\psi} & =\frac{2 n !}{(m+n-1) !} \int_{0}^{\infty} d z e^{-z^{2}} z^{2 m+1} L_{n-1}^{m}\left(z^{2}\right) L_{n-1}^{m}\left(z^{2}\right) \\
& =\frac{2 n !}{2(m+n-1) !} \int_{0}^{\infty} d r e^{-r} r^{m} L_{n-1}^{m}(r) L_{n-1}^{m}(r)=1,
\end{aligned}
$$

so as to have $n=1$ for a typical ground state (corresponding to the hydrogenlike radial quantum number). 


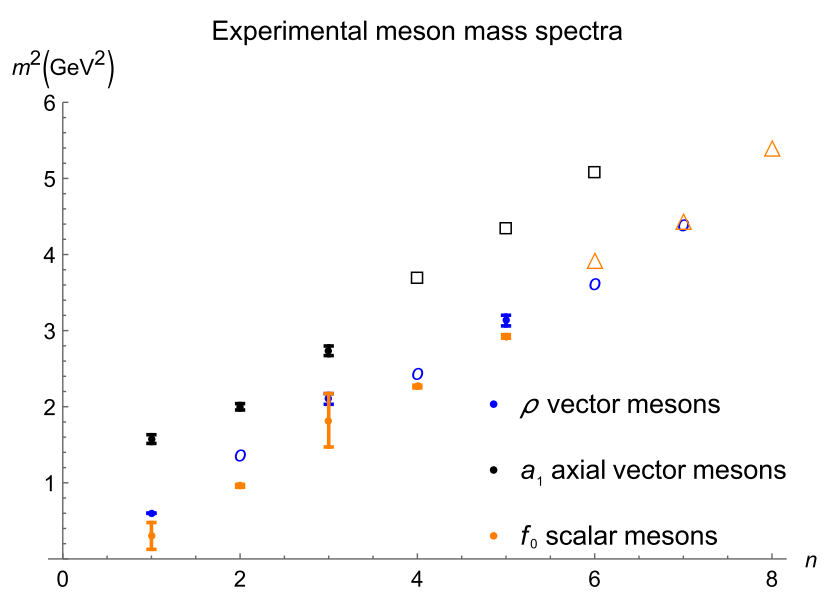

FIG. 1. Experimental mesonic states mass spectra, as a function of the $n$ excitation number. The orange points represent the $f_{0}(500), f_{0}(980), f_{0}(1370), f_{0}(1500), f_{0}(1710)$ scalar mesons. The $f_{0}(2020), f_{0}(2200)$ and $f_{0}(2330)$ states, omitted from the summary table in PDG, are simbolyzed by triangles (" $\Delta$ "). The blue points depict the $\rho(770), \rho(1450), \rho(1700)$ vector meson states, whereas the $\rho^{\prime}(1450), \rho(1570), \rho(1900), \rho(2150)$ and $\rho(2270)$ are depicted by circles ("॰"). The black points represent $a_{1}(1260), a_{1}(1420), a_{1}(1640)$ axial vector mesons and the $a_{1}(1930), a_{1}(2095)$ and $a_{1}(2270)$, omitted from the summary table in PDG, are depicted by empty boxes " $\square$ ". All the experimentally established states have error bars [20].

$$
\begin{gathered}
V_{\mathrm{S}}(z)=\frac{\left(\Phi^{\prime}(z)-3 \mathrm{~A}^{\prime}(z)\right)^{2}-1}{4}-m_{\mathfrak{X}}^{2} e^{2 \mathrm{~A}(z)}, \\
V_{\rho}(z)=\frac{\left(\Phi^{\prime}(z)-\mathrm{A}^{\prime}(z)\right)^{2}-1}{4}, \\
V_{\mathrm{a}}(z)=\frac{\left(\Phi^{\prime}(z)-\mathrm{A}^{\prime}(z)\right)^{2}-1}{4}+\frac{48 \pi^{2}}{N_{c}} e^{2 \mathrm{~A}(z)} \xi^{2}(z) .
\end{gathered}
$$

The potentials (17)-(19), respectively, determine the mass spectra of the $a_{1}, \rho$, and $f_{0}$ meson families, as (squared) eigenvalues in Eqs. (14)-(16). The experimental data for the $a_{1}$ axial vector, the $\rho$ vector, and the $f_{0}$ scalar mesons are shown in Fig. 1. One should also notice that the $\rho$ states depicted in Fig. 1 regard the $\rho(770), \rho(1450)$, and $\rho(1700)$ as confirmed vector meson states in PDG, where between $500 \mathrm{~K}$ and $1.98 \mathrm{M}$ events have been run. On the other hand, the $\rho(1570), \rho(1900), \rho(2150)$, and $\rho(2270)$ are not established particles yet; therefore, they are omitted from the summary table in PDG [20]. In fact, $\rho(1570)$ may be an Okubo-Zweig-Iizuka-violating [53] decay mode of the $\rho(1700)$ state. Together with $\rho(1900), \rho(2150)$, and $\rho(2270)$, they are listed in Ref. [20] as light unflavored mesons, with just 54 events already run. In addition, $f_{0}(500), f_{0}(980), f_{0}(1370), f_{0}(1500)$, and $f_{0}(1710)$ are established scalar meson particles plotted in Fig. 1, whereas $f_{0}(2020), f_{0}(2200)$, and $f_{0}(2330)$ scalar mesonic states are still left out of the summary table in PDG. Finally, the $a_{1}(1260), a_{1}(1420)$, and $a_{1}(1640)$ axial mesons have been experimentally confirmed, whereas the $a_{1}(1930)$, $a_{1}(2095)$, and $a_{1}(2270)$ axial vector mesonic states are also omitted from the summary table in PDG [20], with just a few events registered.

\section{TWO FLAVOR SOFT WALL ADS/QCD IN GRAVITON-DILATON-GLUON BULK}

A two flavor soft wall AdS/QCD can be then considered, where a dilatonic background field is assumed in a chiral and gluon condensate background, with gravity [25]. For the pure gluon system, the scalar glueball CE was already studied in Ref. [8]. In addition, Ref. [19] used two types of dilaton background fields, yielding the glueball spectra in full compliance with lattice data, namely,

$$
\begin{gathered}
\Phi_{1}(z)=\mu_{\mathrm{G}}^{2} z^{2} \\
\Phi_{2}(z)=\mu_{\mathrm{G}}^{2} z^{2} \tanh \left(\frac{\mu_{\mathrm{G}^{2}}^{4} z^{2}}{\mu_{\mathrm{G}}^{2}}\right) .
\end{gathered}
$$

The dual dimension-2 (dimension-4) gluon condensate has a $\mu_{\mathrm{G}}\left(\mu_{\mathrm{G}^{2}}\right)$ energy scale. The $\Phi_{1}(z)$ dilatonic field in Eq. (20) yields the meson spectra and also implements the quarks confinement [17]. It is the object dual to the gluon condensate with dimension-2, meaning the BoseEinstein condensate consisting of strongly coupled paired gluons $[16,21,45]$. The $\Phi_{2}(z)$ dilatonic field in Eq. (21), at the UV regime, behaves as $\lim _{z \rightarrow 0} \Phi_{2}(z)=\mu_{\mathrm{G}^{2}}^{4} z^{4}$, being dual to a gluon condensate that has dimension 4 [54]. At the IR regime, $\lim _{z \rightarrow \infty} \Phi_{2}(z)=\mu_{\mathrm{G}}^{2} z^{2}$. A graviton-dilaton-gluon bulk action can be expressed as a sum of an EinsteinHilbert action for pure gravity in the bulk, an action for gluons written with respect to the $\Phi$ dilaton field, and an action for two flavor bulk mesons on a dilatonic background. This last part of the action implements the dynamics of the $\xi(z)$ scalar field in Eq. (5). The effective graviton-dilaton-gluon bulk action reads [19]

$$
\begin{aligned}
S= & \kappa_{5}^{2} \int \sqrt{-g} e^{-2 \Phi}\left\{\left[R / 4+g_{m n} \partial^{m} \Phi \partial^{n} \Phi-V_{g}(\Phi)\right.\right. \\
& \left.\left.-4 \lambda e^{-\Phi}\left(g_{m n} \partial^{m} \xi \partial^{n} \xi+V(\Phi, \xi)\right)\right]\right\} d^{5} x,
\end{aligned}
$$

where $\lambda$ denotes a general coupling, and $V_{g}$ denotes the gluon system potential. The action (22) yields the following EOM:

$$
\begin{aligned}
& -3 \mathrm{~A}^{\prime \prime}+3 \mathrm{~A}^{\prime 2}+2 \Phi^{\prime \prime}-4 \mathrm{~A}^{\prime} \Phi^{\prime}-2 \lambda e^{\Phi} \xi^{\prime 2}=0, \\
& 8 \Phi^{\prime \prime}+24 \mathrm{~A}^{\prime} \Phi^{\prime}-16 \Phi^{\prime 2}-6 \lambda \xi^{\prime 2} e^{\Phi} \\
& -3 \frac{\partial}{\partial \Phi}\left(\lambda e^{7 \Phi / 3} V(\Phi, \xi)+V_{g}(\Phi)\right) e^{-4 \Phi / 3+2 \mathrm{~A}}=0, \\
& -\xi^{\prime \prime}+\left(\Phi^{\prime}-3 \mathrm{~A}^{\prime}\right) \xi^{\prime}+e^{2 \mathrm{~A}} \frac{\partial^{2} V(\Phi, \xi)}{\partial \xi \partial \Phi}=0 .
\end{aligned}
$$




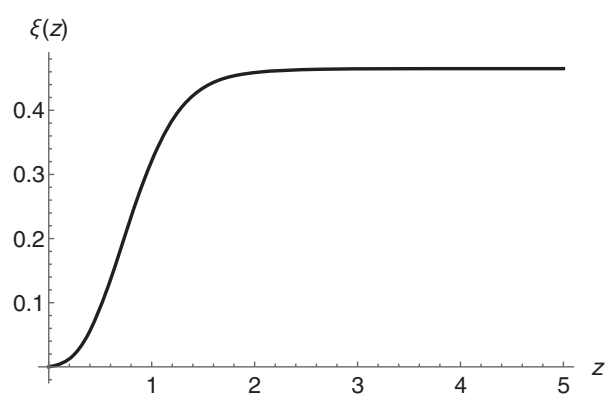

FIG. 2. The $\xi(z)$ field, in the quadratic dilatonic background (20).

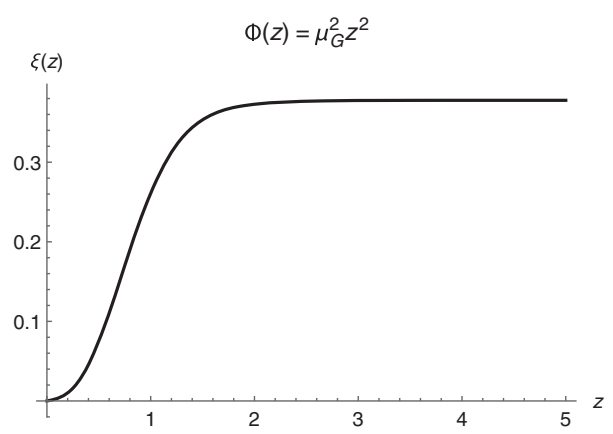

FIG. 3. The $\xi(z)$ field, in the deformed dilatonic background (21).

In what follows $N_{f}$ stands for the number of flavors. In the UV regime, Refs. [19,55] show that $\lim _{z \rightarrow 0} \xi(z)=$ $\frac{m_{q} \tau z}{2}+\frac{\sigma}{2 \tau} z^{3}$, where $m_{q}$ is the quark mass, $\sigma$ denotes the string tension gluing the quark-antiquark condensate, and $\tau^{2}=\frac{N_{c}^{2}}{4 \pi^{2} N_{f}}$, with $N_{c}=3$ and $N_{f}=2$. Reference [19] scrutinized the heavy quark potential under the gravitondilaton-gluon background (22), showing that the potential in $(22)$ and (23c) reads $V(\Phi, \xi) \approx \xi^{2} \Phi^{2}, \lim _{z \rightarrow \infty} \mathrm{A}^{\prime}(z)=0$, and $\lim _{z \rightarrow \infty} \mathrm{A}(z)=a$, for $a$ constant. For both the $\Phi_{1}(z)$ and $\Phi_{2}(z)$, respectively in Eqs. (20) and (21), the parameters $\mu_{\mathrm{G}}=0.43=\mu_{\mathrm{G}^{2}}$ were adopted in Ref. [19], in order to fit the meson spectra data with good accuracy to experimental data. In addition, $\sigma \approx 5.841 \times 10^{6} \mathrm{MeV}$ and $m_{q}=5.81 \mathrm{MeV}$, for the $\Phi_{1}(z)$ quadratic dilaton, whereas $\sigma \approx 4.484 \times 10^{6} \mathrm{MeV}$ and $m_{q}=8.38 \mathrm{MeV}$, for the $\Phi_{2}(z)$ deformed dilaton. Numerical analyses of Eqs. (23a)-(23c) in Ref. [19] yield the solutions for $\xi(z)$, for both the dilatonic backgrounds, in Figs. 2 and 3, in full compliance with the UV and the IR regimes for the dilaton fields in Eqs. (20) and (21).

\section{INFORMATIONAL ENTROPIC REGGE TRAJECTORIES AND MESON MASS SPECTRA}

The meson spectra were computed for the $\Phi_{1}(z)$ and $\Phi_{2}(z)$ dilaton backgrounds in Ref. [19]. Replacing Eqs. (20) and (21), and the scalar field $\xi(z)$ in Eqs. (23a)-(23c), one can derive the $\mathrm{A}(z)$ warp factor. To obtain the $S$ scalar meson
TABLE I. The experimental and predicted mass spectra for $f_{0}$ scalar mesons, in both the quadratic (20) (second column) and the deformed (21) (third column) dilaton profiles, respectively along the rows, for the $f_{0}(500), f_{0}(980), f_{0}(1370), f_{0}(1500)$, $f_{0}(1710), f_{0}(2020), f_{0}(2200)$, and $f_{0}(2330)$ mesons. The modes indicated by asterisks are confirmed states in PDG, whereas the others have not been experimentally confirmed yet [20].

\begin{tabular}{lccr}
\hline \hline \multicolumn{4}{c}{$f_{0}$ scalar meson mass spectra } \\
\hline$n$ & $\begin{array}{c}\text { Experiment } \\
(\mathrm{MeV})\end{array}$ & $\begin{array}{c}\operatorname{Mass}_{\Phi_{1}(z)} \\
(\mathrm{MeV})\end{array}$ & $\begin{array}{c}\operatorname{Mass}_{\Phi_{2}(z)} \\
(\mathrm{MeV})\end{array}$ \\
\hline $1^{*}$ & $563_{-69}^{+58}$ & 420.9 & 186.9 \\
$2^{*}$ & $990 \pm 20$ & 1042.6 & 1077.8 \\
$3^{*}$ & $1400 \pm 40$ & 1369.5 & 1434.0 \\
$4^{*}$ & $1504 \pm 6$ & 1625.0 & 1684.5 \\
$5^{*}$ & $1723_{-5}^{+6}$ & 1842.4 & 1889.7 \\
6 & $1992 \pm 16$ & 2035.7 & 2067.4 \\
7 & $2189 \pm 13$ & 2211.9 & 2233.8 \\
8 & $2337 \pm 14$ & 2374.8 & 2391.8 \\
\hline \hline
\end{tabular}

mass spectra, the following action was employed in Ref. [19]:

$$
S_{s}=\kappa \int e^{-\Phi} \sqrt{-g}\left(\partial_{m} S \partial^{m} S+2 S^{2} \Phi^{2}\right) d^{5} x
$$

where $\partial_{m} S \partial^{m} S=\partial_{z} S \partial^{z} S+\partial_{\mu} S \partial^{\mu} S$ and $\kappa=-2 \frac{N_{f}}{N_{c} \ell^{3}}$. The EOM for the $S$ scalar is given by Eq. (14), however with the Schrödinger potential

$$
\begin{aligned}
V_{\mathrm{S}}(z)= & \frac{1}{4}\left(\Phi^{\prime}-3 \mathrm{~A}^{\prime}\right)^{2}+\frac{3}{2}\left(3 \mathrm{~A}^{\prime \prime}-\Phi^{\prime \prime}\right) \\
& -\left(2 \mathrm{~A}^{\prime}-\frac{\Phi^{\prime}}{2}+\frac{\Phi^{\prime}}{2(1+\Phi)}\right)\left(3 \mathrm{~A}^{\prime}-\Phi^{\prime}\right) \\
& +\left(\mathrm{A}^{\prime}-\frac{3}{2} \Phi^{\prime}-\frac{\Phi^{\prime}}{2(1+\Phi)}\right) \log \left(2 \xi^{\prime}\right)^{\prime}+\frac{\xi^{\prime \prime \prime}}{\xi^{\prime}}
\end{aligned}
$$

instead. The mass spectra for the $f_{0}$ scalar meson family is then obtained, and it is listed in the second and third rows of Table I, respectively, for the quadratic [Eq. (20)] and the deformed [Eq. (21)] dilaton fields. This is accomplished by solving Eq. (14) with the potential in Eq. (25), using the boundary condition $\lim _{z \rightarrow \infty} \mathrm{s}_{n}{ }^{\prime}(z)=0$ and $\mathrm{s}_{n}(0)=0$, with parameters $m_{q} \approx 9 \mathrm{MeV}$ and $\mu \approx 429 \mathrm{MeV}$. The first column replicates the mass spectra in the PDG 2018 for $f_{0}(500), f_{0}(980), f_{0}(1370), f_{0}(1500), f_{0}(1710)$, as well as for the $f_{0}(2020), f_{0}(2200)$, and $f_{0}(2330)$ scalar mesonic states, which are still omitted from the summary table in PDG (few events registered [20]). For deriving the mass spectra for the $\rho$ meson family, for $\kappa=\frac{N_{f}}{2 g_{5}^{2} N_{c} \ell^{3}}$, the action

$$
S_{V}=-\kappa \int e^{5 \mathrm{~A}-\Phi} g_{\mu \nu} \partial_{m} V_{\mu}^{\top} \partial^{m} V^{\top \nu} d^{5} x
$$


TABLE II. The experimental [20] and predicted mass spectra for the $\rho$ vector meson family, in both the quadratic [Eq. (20)] and the deformed [Eq. (21)] dilaton profiles, respectively along the rows, for the $\rho(770), \rho^{\prime}(1450), \rho(1450), \rho(1570), \rho(1700)$, $\rho(1900), \rho(2150)$, and $\rho(2270)$ mesons. The modes indicated by asterisks are experimentally confirmed states, whereas the others are omitted from the summary table in PDG [20].

\begin{tabular}{lccc}
\hline \multicolumn{4}{c}{$\rho$ vector mesons mass spectra } \\
\hline$n$ & $\begin{array}{c}\text { Experiment } \\
(\mathrm{MeV})\end{array}$ & $\begin{array}{c}\text { Mass }_{\Phi_{1}(z)} \\
(\mathrm{MeV})\end{array}$ & $\begin{array}{c}\text { Mass }_{\Phi_{2}(z)} \\
(\mathrm{MeV})\end{array}$ \\
\hline $1^{*}$ & $775.26 \pm 0.25$ & 727.8 & 753.9 \\
2 & $1350_{-50}^{+40}$ & 1134.6 & 1133.8 \\
$3^{*}$ & $1465 \pm 25$ & 1426.0 & 1430.0 \\
4 & $1570 \pm 98$ & 1534.1 & 1537.9 \\
$5^{*}$ & $1720 \pm 20$ & 1664.5 & 1667.9 \\
6 & $1909 \pm 30$ & 1873.6 & 1875.4 \\
7 & $2149 \pm 17$ & 2061.9 & 2063.7 \\
8 & $2265 \pm 40$ & 2233.6 & 2234.6 \\
\hline \hline
\end{tabular}

is employed, where $V_{m}^{\top}$ denotes the transverse components in Eq. (17). The normalizable solutions $\mathrm{v}_{n}$ of the associated EOMs (15) and (18) are obtained as Kaluza-Klein modes for discrete values of the $4 \mathrm{~d}$ momentum $q^{2}=m_{n}^{2}$. The boundary fields $\mathrm{V}_{n}$ [cf. Eq. (15)] play the role of external sources, coupled to the QCD current densities. The $\rho$ meson family mass spectra can then be obtained from Eq. (15) with the boundary condition $\mathrm{v}_{n}(0)=0, \lim _{z \rightarrow \infty} \mathrm{v}_{n}{ }^{\prime}(z)=0$. The mass spectra are shown in the second and third rows of Table II, respectively, for quadratic [Eq. (20)] and deformed [Eq. (21)] dilaton fields [19]. The first column in Table II corresponds to the experimental data in Fig. 1. Similarly to the $\rho$ mesons, the $a_{n}$ axial vector mesonic excitations [cf. Eq. (16)] describe the $a_{1}$ axial vector meson family, whose mass spectra can be obtained from the modes of the axial gauge field (6b) in the bulk. The quadratic terms in the transverse component of the axial vector $\stackrel{\circ}{m}^{m}$ (6b), denoted by $\stackrel{\circ}{A}^{\top}$, are used to construct the action

$$
S_{\AA}=\nprec \int e^{5 \mathrm{~A}-\Phi}\left(\partial_{m} \stackrel{\circ}{A}_{\mu}^{\top} \partial^{m} \stackrel{\circ}{A}^{\top \mu}+\frac{4 g_{5}^{2} \xi^{2}}{\ell^{2}} \stackrel{\circ}{A}_{\mu}^{\top} \stackrel{\circ}{A}{ }^{\top \mu}\right) d^{5} x,
$$

where $g_{5}^{2}=4 \pi^{2} N_{f} / N_{c}$. The bulk effective mass in (27) is generated by the Higgs mechanism, with the $\mathfrak{X}$ scalar field encoding the chiral symmetry breaking [56]. The EOMs that drive the axial vector mesonic states can be written as Eqs. (16)-(19), with boundary conditions $E \lim _{z \rightarrow \infty} \mathrm{a}_{n}^{\prime}(z)=0$ and $\mathrm{a}_{n}(0)=0$. The mass spectra of the $a_{1}$ axial vector meson family are shown in the second and third rows of Table III, respectively, for quadratic [Eq. (20)] and deformed [Eq. (21)] dilaton fields. The first column in Table III represents the experimental data [20]. For the $a_{1}(1260), a_{1}(1420)$, and $a_{1}(1640)$ axial mesons
TABLE III. The experimental [20] and predicted mass spectra for $a_{1}$ axial vector mesons, in both the quadratic [Eq. (20)] and the deformed [Eq. (21)] dilaton profiles, respectively along the rows, for $a_{1}(1260), a_{1}(1420), a_{1}(1640), a_{1}(1930), a_{1}(2095)$, and $a_{1}(2270)$ mesons. The modes indicated by asterisks are confirmed states in PDG [20].

\begin{tabular}{lccc}
\hline \hline \multicolumn{4}{c}{$a_{1}$ meson mass spectra } \\
\hline$n$ & $\begin{array}{c}\text { Experiment } \\
(\mathrm{MeV})\end{array}$ & $\begin{array}{c}\text { Mass }_{\Phi_{1}(z)} \\
(\mathrm{MeV})\end{array}$ & $\begin{array}{c}\text { Mass }_{\Phi_{2}(z)} \\
(\mathrm{MeV})\end{array}$ \\
\hline $1^{*}$ & $1255_{-23}^{+13}$ & 1064.9 & 1117.5 \\
$2^{*}$ & $1414_{-13}^{+15}$ & 1364.4 & 1624.1 \\
$3^{*}$ & $1654 \pm 19$ & 1561.7 & 1623.0 \\
4 & $1930_{-70}^{+30}$ & 1845.8 & 1878.6 \\
5 & $2096 \pm 138$ & 2059.0 & 2082.5 \\
6 & $2265 \pm 50$ & 2242.0 & 2262.7 \\
\hline \hline
\end{tabular}

experimentally confirmed, as well as for $a_{1}(1930)$, $a_{1}(2095)$, and $a_{1}(2270)$, the axial vector mesonic states are omitted from the summary table in PDG [20] (few events registered). Now, in order to compute the $\mathrm{CE}$ for the $f_{0}$, the $\rho$, and the $a_{1}$ meson families, one first considers a localized, Lebesgue-integrable $\epsilon(z)$ energy density, associated with each meson family. In general, given an arbitrary Lagrangian $\mathfrak{Q}$, the energy-momentum tensor reads

$$
T^{m n}=\frac{2}{\sqrt{-g}}\left(\frac{\partial(\sqrt{-g} \mathfrak{Q})}{\partial g_{m n}}-\partial_{x^{q}} \frac{\partial(\sqrt{-g} \mathfrak{Q})}{\partial\left(\frac{\partial g_{m n}}{\partial x^{q}}\right)}\right) .
$$

The $\epsilon(z)$ energy density corresponds to the $T_{00}(z)$ component of (28), respectively, for the $f_{0}$ meson family (24), for the $\rho$ meson family (26), and for the $a_{1}$ meson family (27). The Fourier transform $\epsilon(k)=\int_{\mathbb{R}} \epsilon(z) e^{-i k \cdot z} d z$, with respect to the $z$ dimension that defines the energy scale in AdS/ $\mathrm{QCD}$, is then employed to define the modal fraction $[3,6]$

$$
\epsilon(k)=\frac{|\epsilon(k)|^{2}}{\int_{\mathbb{R}}|\epsilon(k)|^{2} d k} .
$$

It is a correlation probability distribution that quantifies how much a $k$ wave mode contributes to the power spectrum, associated with the energy density. The CE, then, measures the information content of the spatial profile that characterizes the energy density $\epsilon(z)$, with respect to the Fourier wave modes. The CE is defined by $[3,6]$

$$
S[\epsilon]=-\int_{\mathbb{R}} \epsilon_{\diamond}(k) \log \epsilon_{\diamond}(k) d k,
$$

for $\epsilon_{\diamond}(k)=\epsilon(k) / \epsilon_{\max }(k)$. After numerical calculations, the $\mathrm{CE}$ is obtained as a function of the $n$ excitation number, $1 \leq n \leq 8$, for the $a_{1}$ axial vector, the $\rho$ vector, and the $f_{0}$ scalar mesons. The results are listed in Tables IV and V, respectively, for the quadratic and deformed dilatonic field backgrounds. In both Tables IV and V, respectively along 
TABLE IV. The CE for the $\rho$ vector, the $a_{1}$ axial vector, and the $f_{0}$ scalar meson families, in the $\Phi_{1}(z)=\mu_{\mathrm{G}}^{2} z^{2}$ quadratic dilaton soft wall model.

\begin{tabular}{lccc}
\hline \hline \multicolumn{3}{c}{$\Phi_{1}(z)=\mu_{\mathrm{G}}^{2} z^{2}$} \\
\hline$n$ & $\rho$ mesons CE & $a_{1}$ mesons CE & $f_{0}$ mesons CE \\
\hline 1 & 32.8 & 338.2 & 3.26 \\
2 & 307.2 & $1.612 \times 10^{3}$ & 34.81 \\
3 & $2.108 \times 10^{3}$ & $1.027 \times 10^{4}$ & 222.4 \\
4 & $9.832 \times 10^{3}$ & $5.804 \times 10^{4}$ & 913.1 \\
5 & $4.902 \times 10^{4}$ & $2.421 \times 10^{5}$ & $4.723 \times 10^{3}$ \\
6 & $2.092 \times 10^{5}$ & $1.614 \times 10^{6}$ & $2.688 \times 10^{4}$ \\
7 & $7.721 \times 10^{5}$ & $1.057 \times 10^{7}$ & $1.571 \times 10^{5}$ \\
8 & $3.423 \times 10^{6}$ & $2.762 \times 10^{7}$ & $5.241 \times 10^{5}$ \\
\hline \hline
\end{tabular}

TABLE V. The CE for the $\rho$ vector, the $a_{1}$ axial vector, and the $f_{0}$ scalar meson families, in the $\Phi_{2}(z)=z^{2} \tanh \left(\frac{\mu_{\mathrm{G}^{2}}^{4} z^{2}}{\mu_{\mathrm{G}}^{2}}\right)$ deformed dilaton soft wall model.

\begin{tabular}{lccc}
\hline \hline \multicolumn{4}{c}{$\Phi_{2}(z)=\mu_{\mathrm{G}}^{2} z^{2} \tanh \left(\mu_{\mathrm{G}^{2}}^{4} z^{2} / \mu_{\mathrm{G}}^{2}\right)$} \\
\hline$n$ & $\rho$ mesons CE & $a_{1}$ mesons CE & $f_{0}$ mesons CE \\
\hline 1 & 28.05 & 587.51 & 4.28 \\
2 & 547.49 & $2.621 \times 10^{3}$ & 44.32 \\
3 & $2.223 \times 10^{3}$ & $1.518 \times 10^{4}$ & 288.31 \\
4 & $1.016 \times 10^{4}$ & $7.142 \times 10^{4}$ & $1.528 \times 10^{3}$ \\
5 & $5.966 \times 10^{4}$ & $2.982 \times 10^{5}$ & $8.984 \times 10^{3}$ \\
6 & $3.417 \times 10^{5}$ & $1.728 \times 10^{6}$ & $5.347 \times 10^{4}$ \\
7 & $2.017 \times 10^{5}$ & $7.135 \times 10^{6}$ & $2.491 \times 10^{5}$ \\
8 & $5.822 \times 10^{6}$ & $3.231 \times 10^{7}$ & $1.167 \times 10^{6}$ \\
\hline \hline
\end{tabular}

the rows, the first column is depicted for the $\rho$ vector meson family, identifying the $\mathrm{v}_{n}$ eigenfunctions in Eq. (15) as $\mathrm{v}_{1}=\rho(770), \mathrm{v}_{2}=\rho^{\prime}(1450), \mathrm{v}_{3}=\rho(1450), \mathrm{v}_{4}=\rho(1570)$, $\mathrm{v}_{5}=\rho(1700), \mathrm{v}_{6}=\rho(1900), \mathrm{v}_{7}=\rho(2150)$, and $\mathrm{v}_{8}=$ $\rho(2270)$. The second columns in Tables IV and $\mathrm{V}$ are composed of the $a_{n}$ eigenfunctions in Eq. (16), describing the $a_{1}$ axial vector meson family by the identification $a_{1}=$ $a_{1}(1260), \mathrm{a}_{2}=a_{1}(1420), \mathrm{a}_{3}=a_{1}(1640), \mathrm{a}_{4}=a_{1}(1930)$, $\mathrm{a}_{5}=a_{1}(2095)$, and $\mathrm{a}_{6}=a_{1}(2270)$. The mesonic excitations $\mathrm{a}_{7}$ and $\mathrm{a}_{8}$ are solutions of the $\operatorname{EOM}(14)$, for $n=7$ and $n=8$, not detected yet. In addition, the third columns in Tables IV and V show the $f_{0}$ scalar meson family, described by the $s_{n}$ wave eigenfunctions of Eq. (14). They read $\mathrm{s}_{1}=f_{0}(500), \mathrm{s}_{2}=f_{0}(980), \mathrm{s}_{3}=f_{0}(1370), \mathbf{s}_{4}=f_{0}(1500)$, $\mathrm{s}_{5}=f_{0}(1710), \mathbf{s}_{6}=f_{0}(2020), \mathbf{s}_{7}=f_{0}(2200)$, and $\mathbf{s}_{8}=$ $f_{0}(2330)$. First, analyzing the quadratic dilatonic potential in Eq. (20), one takes the $\mathrm{CE}$ for the $\rho, a_{1}$, and $f_{0}$ meson families listed in Table IV. Computing the logarithm of the CE for each $n$ mode for the three meson families leads to the result depicted in Fig. 4, whose numerical interpolation provides the first type of informational entropic Regge trajectories. For the $\rho$ vector, the $a_{1}$ axial vector, and the $f_{0}$

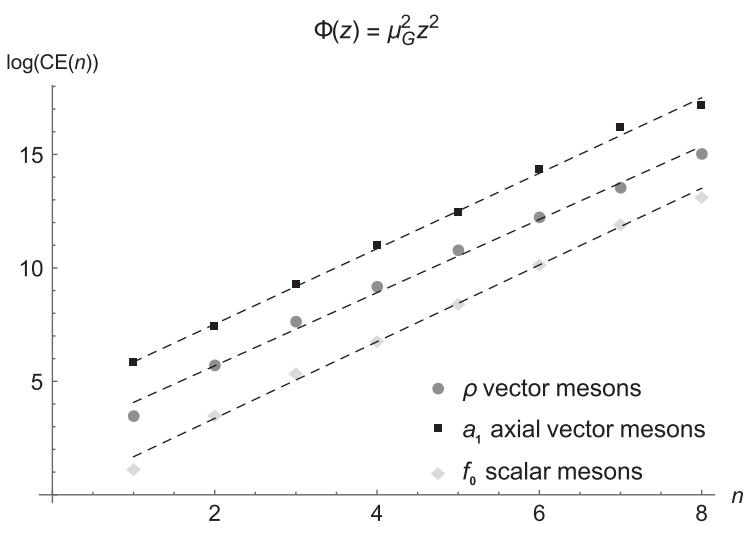

FIG. 4. Logarithm of the configurational entropy of mesons, the families of $\rho, a_{1}$, and $f_{0}$ mesons, in the quadratic dilaton soft wall model.

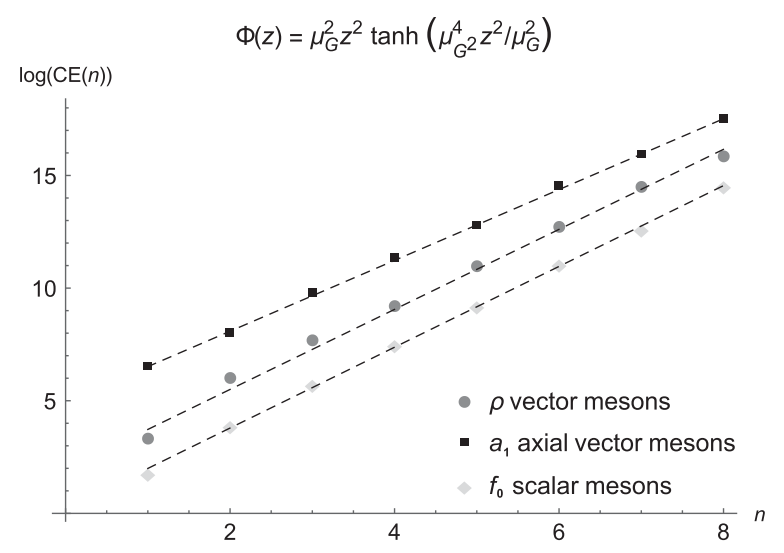

FIG. 5. Logarithm of the configurational entropy of the families of $\rho, a_{1}$, and $f_{0}$ mesons, in the deformed soft wall AdS/QCD.

scalar meson families, respectively, the informational entropic Regge trajectories are the dotted lines in Fig. 4. Their explicit expressions are, respectively,

$$
\begin{aligned}
& \log \left(\mathrm{CE}_{\rho}(n)\right)=1.6123 n+2.4594, \\
& \log \left(\mathrm{CE}_{a_{1}}(n)\right)=1.6632 n+4.1923, \\
& \log \left(\mathrm{CE}_{f_{0}}(n)\right)=1.6907 n+0.0150,
\end{aligned}
$$

within $0.9 \%, 1.2 \%$, and $\sim 1.7 \%$ standard deviations, respectively. Now, with the computed CE for the $\rho, a_{1}$, and $f_{0}$ meson families in the $\Phi_{2}$ dilaton background (21), listed in Table V, one can also calculate the logarithm of the CE, for each $n$ excitation mode. Figure 5 shows the corresponding results for each meson family, wherein linear regression yields the second type of informational entropic Regge trajectories. The explicit expressions for each informational entropic Regge trajectory are numerically obtained by interpolation of the data in Table V. The obtained linear regressions, respectively for the $a_{1}$ axial vector, the $\rho$ vector, and the $f_{0}$ scalar meson families, read 


$$
\begin{aligned}
& \log \left(\mathrm{CE}_{\rho}(n)\right)=1.8497 n+1.7763, \\
& \log \left(\mathrm{CE}_{a_{1}}(n)\right)=1.5623 n+4.9425, \\
& \log \left(\mathrm{CE}_{f_{0}}(n)\right)=1.7624 n+0.2112,
\end{aligned}
$$

within $\sim 0.6 \%, \sim 1.4 \%$, and $\sim 1.7 \%$ standard deviations, respectively. For the $\rho, a_{1}$, and $f_{0}$ meson families, one can hence realize a scaling law, relating the logarithm of the CE and the $n$ meson excitation modes. Figures 4 and 5 show that there are informational entropic Regge trajectories, implementing a relation between the logarithm of the $\mathrm{CE}$ and the $n$ excitation number, for both the quadratic and deformed dilatonic potentials. The original Regge trajectories in the soft wall AdS/QCD regard the relation $m_{n} \sim n$, for the lightflavor meson mass spectra. One can then emulate them in the information entropic context. In fact, one can calculate the logarithm of the CE for each meson family, as a function of the meson mass spectra, experimentally detected. In what follows, the $\Phi_{2}(z)=z^{2} \tanh \left(\frac{\mu_{\mathrm{G}^{2}}^{4} z^{2}}{\mu_{\mathrm{G}}^{2}}\right)$ deformed dilaton in the soft wall AdS/QCD model is employed, as it better describes the meson mass spectra. The results are plotted in Fig. 6. The informational entropic Regge trajectories, as a function of the meson mass $m(\mathrm{MeV})$, are, respectively, listed as follows:

$$
\begin{aligned}
& \log \left(\mathrm{CE}_{\rho}(m)\right)=0.0067 m-1.9651, \\
& \log \left(\mathrm{CE}_{a_{1}}(m)\right)=0.0069 m-2.2184, \\
& \log \left(\mathrm{CE}_{f_{0}}(m)\right)=0.0062 m-2.2013,
\end{aligned}
$$

within $\sim 2.6 \%, \sim 2.1 \%$, and $\sim 2.5 \%$ standard deviations, respectively. The informational entropic Regge trajectories in Eqs. (37)-(39) provide another very interesting aspect of the $\mathrm{CE}$ that underlies the meson families. Instead of computing the meson family mass spectra, solving Eqs. (14)-(16), one can extrapolate the interpolation lines (34)-(36) to compute the CE for the meson families, at least for the $n$ mesonic excitations such that $n>8$ for the $\rho$ and $f_{0}$ families and such that $n>6$ for the $a_{1}$ family.

In the following discussion of the informational entropic Regge trajectories (34)-(36), the notation $m_{\alpha, n}$ means the mass of the $n$th meson in some $\alpha$-meson family $\left(\alpha=\rho, a_{1}, f_{0}\right)$, corresponding to the element in the respective meson family with $n$ excitation number. Considering the experimental value of the $\rho$ vector meson family, although there is no mesonic excitation $\mathrm{v}_{n}$ in the $\rho$ meson family higher than $n=8$, experimentally detected, the informational entropic Regge trajectory in Fig. 6 deploys a reliable method for predicting the mass of the $\mathrm{v}_{n}$ vector meson states, for $n \geq 9$. Although the explicit calculation by Eq. (15), with potential (18), is already known to produce the mass spectra of the $\rho$ vector meson family as a function of the $n$ excitation
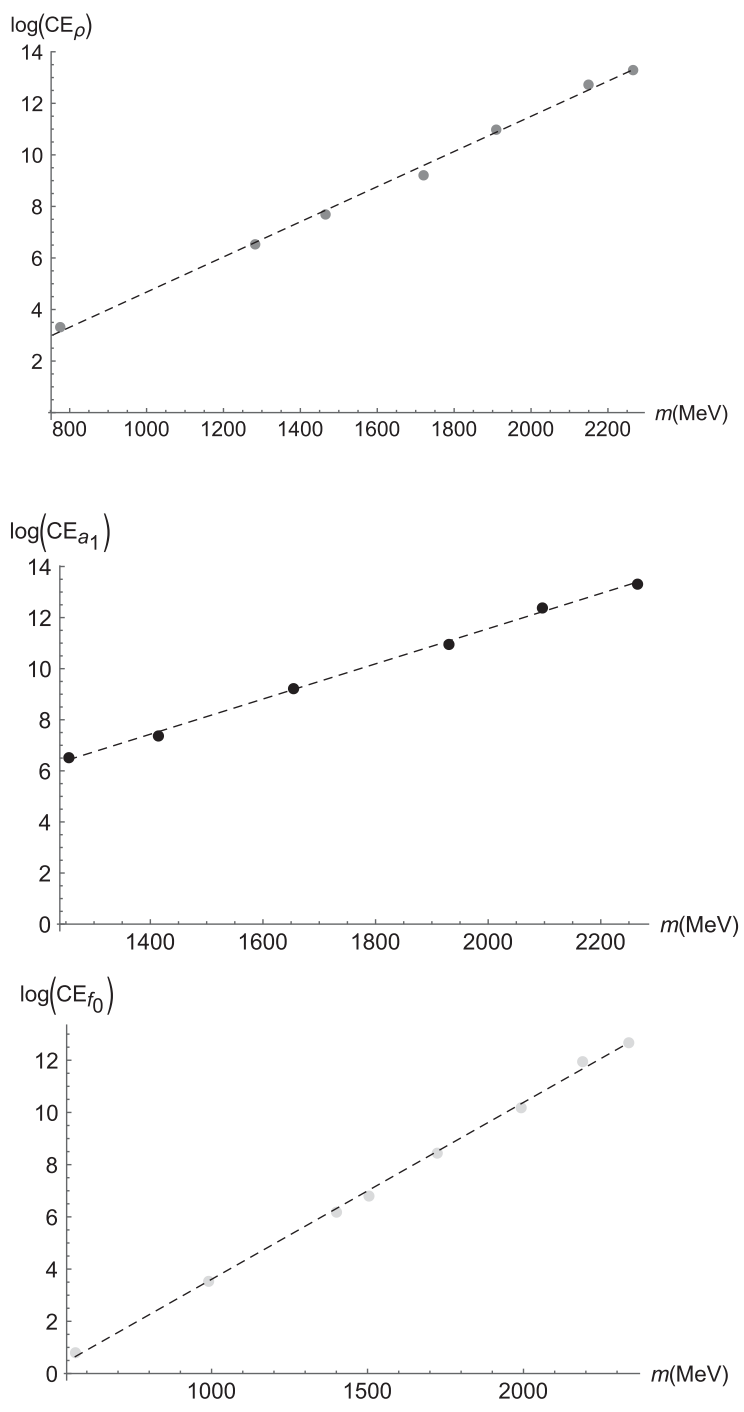

FIG. 6. Logarithm of the configurational entropy of the $\rho$ (blue), $a_{1}$ (orange), and $f_{0}$ (green) meson families, as a function of their mass spectra. The respective informational entropic Regge trajectories are also plotted.

number, here the masses of the $\mathrm{v}_{9}$ and $\mathrm{v}_{10}$ elements in the $\rho$ meson mass family can be inferred. In fact, for $n=9$, Eq. (34) yields $\log \left(\mathrm{CE}_{\rho}\right)=18.423$. Replacing this value in the informational entropic Regge trajectory (37), one obtains the mass $m_{\rho, 9}=2878 \mathrm{MeV}$, for the $\mathrm{v}_{9}$ mesonic state in the $\rho$ meson family. The standard deviations for Eqs. (34)-(37) give the reliable range $2813 \mathrm{MeV} \lesssim m_{\rho, 9} \lesssim 2945 \mathrm{MeV}$ for the $\mathrm{v}_{9}$ vector meson state. Similarly, the $\mathrm{v}_{10}$ vector meson excitation has mass $m_{\rho, 10}=3098 \mathrm{MeV}$. Considering the standard deviations for Eqs. (34)-(37), the range $3042 \lesssim$ $m_{\rho, 10} \lesssim 3153 \mathrm{MeV}$ is a reliable one. One can further extrapolate the mass spectra for the $\mathrm{v}_{n}$ vector mesonic excitations for $n \geq 11$; however, the standard deviations are larger, the higher the $n$ excitation number is. Analogously, the $a_{1}$ axial vector meson family can be analyzed. The masses of the $a_{7}$ and $a_{8}$ can then be inferred, using the mass 
spectra of the experimentally detected mesons in this family, with Eq. (35)-(38). In fact, for $n=7$, Eq. (35) yields $\log \left(\mathrm{CE}_{a_{1}}\right)=15.878$. Replacing this value in the informational entropic Regge trajectory (37), one obtains the mass $m_{a_{1}, 7}=2567 \mathrm{MeV}$ for the $a_{7}$ mesonic state in the $a_{1}$ meson family. The standard deviations for Eqs. (35) and (38) give the reliable range $2491 \mathrm{MeV} \lesssim m_{a_{1}, 7} \lesssim 2637 \mathrm{MeV}$ for the $\mathrm{a}_{7}$ axial vector meson state. Similarly, the $\mathrm{a}_{8}$ vector meson excitation has mass $m_{a_{1}, 8}=2782 \mathrm{MeV}$. The standard deviations for Eqs. (34) and (37) yield the range $2708 \lesssim$ $m_{a_{1}, 8} \lesssim 2869 \mathrm{MeV}$. One can further infer the mass spectra of the $\mathrm{a}_{n}$ vector mesonic excitations for $n \geq 9$. Finally, the masses of the next generation of $f_{0}$ scalar mesons can be predicted. Employing Eq. (36) for $n=9$ implies that $\log \left(\mathrm{CE}_{f_{0}}\right)=16.073$. Now, one can substitute this value into Eq. (39), yielding the mass of the $\mathrm{s}_{9}$ element in the $f_{0}$ meson family, $m_{f_{0}, 9}=2905 \mathrm{MeV}$. The standard deviations for Eqs. (36)-(39) give the reliable range $2806 \mathrm{MeV} \lesssim$ $m_{f_{0}, 9} \lesssim 2996 \mathrm{MeV}$ for the $\mathrm{s}_{9}$ scalar mesonic excitation. In addition, similar calculations yield the $s_{10}$ scalar meson excitation mass, $m_{f_{0}, 10}=3189 \mathrm{MeV}$. The standard deviations related to Eqs. (34) and (37) imply the range $3094 \lesssim$ $m_{\rho, 10} \lesssim 3301 \mathrm{MeV}$ for the $\mathrm{s}_{10}$ scalar meson. One can further extrapolate the mass spectra of the $s_{n}$ scalar mesonic excitations for $n \geq 11$; however, the standard deviations increase as the $n$ excitation number gets higher.

\section{CONCLUDING REMARKS AND PERSPECTIVES}

The CE was computed for the $a_{1}$ axial vector, the $\rho$ vector, and the $f_{0}$ scalar meson families for two dilatonic backgrounds in a graviton-dilaton-gluon background. Two types of informational entropic Regge trajectories were derived for each meson family. The first one consists of the $\mathrm{CE}$ in terms of the meson $n$ excitation number, described by Eqs. (34)-(36) and illustrated in Figs. 4 and 5, for both quadratic and deformed dilatonic profiles. The second type of informational entropic Regge trajectory relates the logarithm of the CE to the experimental mass spectra of the meson families in Eqs. (37)-(39), respectively shown in the plots of Fig. 6. Consequently, the meson family mass spectra were extrapolated from these informational entropic Regge trajectories. A range for the mass spectra of mesons with higher $n$ excitation numbers, in each meson family, was then estimated with good accuracy. The first two elements of the next generation, in each meson family, were studied and discussed.

The prediction of the meson mass spectra through Eqs. (14)-(16), although also taking experimental parameters to fit the meson mass spectra, is a theoretical prediction that already matches experimental data, as shown in Ref. [19] and illustrated in Tables I-III. The very essence of the procedure throughout Sec. IV estimates the mass spectra of the next generation of mesons by the informational entropic Regge trajectories based on the mass spectra of the already detected mesons. Indeed, the first types (34)-(36) of informational entropic Regge trajectories express the CE once the $n$ excitation number is fixed. With the obtained value of the CE, Eqs. (37)-(39) then determine the values of the masses of the next generation of mesonic states in each $\rho, a_{1}$, and $f_{0}$ family. Since the informational entropic Regge trajectories are determined by the experimental meson mass spectra, this procedure can determine at least the next two elements in each meson family with good accuracy. The eventual detection of these new mesonic states shall contribute to more experimental points in the plots of Fig. 6, improving the fitting of Eqs. (37)-(39).

We can further regard pseudoscalar mesons whose CE may also be computed. However, their Lagrangian involves a pseudoscalar field that is coupled to a $\varphi$ scalar field that defines the parallel axial vector [complementary to the transverse to the $\stackrel{\circ}{A}_{\mu}^{\top}$ field in (24)] as $\partial_{\mu} \varphi$. Hence, the derived coupled system of EOMs involves awkward Schrödingerlike potentials, turning the CE computation into a difficult task, which is unsolved up to now. In addition, as the soft wall AdS/QCD model corresponds to the $D_{3}-D_{q}$, system, extensions involving $D_{p}-D_{q}$ models [57] may also be accomplished, although it is far beyond the scope assumed here. Finite temperature effects in the soft wall AdS/QCD may also be implemented; their initial results using the $\mathrm{CE}$ apparatus were introduced in Ref. [58] for quarkonia.

\section{ACKNOWLEDGMENTS}

The work of A.E. B. is supported by the Brazilian Agencies FAPESP (Grant No. 2018/03960-9) and CNPq (Grant No. 300831/2016-1). R. d. R. is grateful to FAPESP (Grant No. 2017/18897-8) and to the National Council for Scientific and Technological Development-CNPq (Grant No. 303293/2015-2) for partial financial support.
[1] C. E. Shannon, Bell Syst. Tech. J. 27, 379 (1948).

[2] M. Gleiser and N. Stamatopoulos, Phys. Lett. B 713, 304 (2012).

[3] M. Gleiser and N. Stamatopoulos, Phys. Rev. D 86, 045004 (2012).
[4] M. Gleiser, M. Stephens, and D. Sowinski, Phys. Rev. D 97, 096007 (2018).

[5] M. Gleiser and N. Graham, Phys. Rev. D 89, 083502 (2014).

[6] M. Gleiser and D. Sowinski, Phys. Lett. B 747, 125 (2015). 
[7] A. E. Bernardini and R. da Rocha, Phys. Lett. B 762, 107 (2016).

[8] A. E. Bernardini, N. R. F. Braga, and R. da Rocha, Phys. Lett. B 765, 81 (2017).

[9] N. R. F. Braga and R. da Rocha, Phys. Lett. B 776, 78 (2018).

[10] D. Sowinski and M. Gleiser, J. Stat. Phys. 167, 1221 (2017).

[11] N. R. F. Braga and R. da Rocha, Phys. Lett. B 767, 386 (2017).

[12] E. Witten, arXiv:1805.11965.

[13] M. Natsuume, Lect. Notes Phys. 903, 1 (2015).

[14] J. Polchinski and M. J. Strassler, Phys. Rev. Lett. 88, 031601 (2002).

[15] H. Boschi-Filho and N. R. F. Braga, Eur. Phys. J. C 32, 529 (2004).

[16] C. Csaki and M. Reece, J. High Energy Phys. 05 (2007) 062.

[17] A. Karch, E. Katz, D. T. Son, and M. A. Stephanov, Phys. Rev. D 74, 015005 (2006).

[18] S. J. Brodsky, G. F. de Teramond, H. G. Dosch, and J. Erlich, Phys. Rep. 584, 1 (2015).

[19] D. Li and M. Huang, J. High Energy Phys. 11 (2013) 088.

[20] M. Tanabashi et al. (Particle Data Group), Phys. Rev. D 98, 030001 (2018).

[21] T. Gherghetta, J. I. Kapusta, and T. M. Kelley, Phys. Rev. D 79, 076003 (2009).

[22] L. Da Rold and A. Pomarol, Nucl. Phys. B721, 79 (2005).

[23] P. Zhang, J. High Energy Phys. 05 (2010) 039.

[24] Y.-Q. Sui, Y.-L. Wu, Z.-F. Xie, and Y.-B. Yang, Phys. Rev. D 81, 014024 (2010).

[25] P. Colangelo, F. Giannuzzi, S. Nicotri, and V. Tangorra, Eur. Phys. J. C 72, 2096 (2012).

[26] G. 't Hooft, Nucl. Phys. B75, 461 (1974).

[27] P. Colangelo and F. Loparco, arXiv:1811.05272.

[28] N. Barbosa-Cendejas, R. Cartas-Fuentevilla, A. HerreraAguilar, R. R. Mora-Luna, and R. da Rocha, Phys. Lett. B 782, 607 (2018).

[29] N. R. F. Braga, L. F. Ferreira, and R. da Rocha, Phys. Lett. B 787, 16 (2018).

[30] A. Goncalves and R. da Rocha, Phys. Lett. B 774, 98 (2017).

[31] G. Karapetyan, Phys. Lett. B 781, 201 (2018).

[32] G. Karapetyan, Phys. Lett. B 786, 418 (2018).

[33] G. Karapetyan, Europhys. Lett. 117, 18001 (2017).
[34] G. Karapetyan, Europhys. Lett. 118, 38001 (2017).

[35] C. W. Ma and Y. G. Ma, Prog. Part. Nucl. Phys. 99, 120 (2018).

[36] M. Gleiser and D. Sowinski, Phys. Lett. B 727, 272 (2013).

[37] M. Gleiser and N. Jiang, Phys. Rev. D 92, 044046 (2015).

[38] R. Casadio and R. da Rocha, Phys. Lett. B 763, 434 (2016).

[39] R. A. C. Correa and R. da Rocha, Eur. Phys. J. C 75, 522 (2015).

[40] R. A. C. Correa, D. M. Dantas, C. A. S. Almeida, and R. da Rocha, Phys. Lett. B 755, 358 (2016).

[41] A. Alves, A. G. Dias, and R. da Silva, Braz. J. Phys. 47, 426 (2017).

[42] A. Alves, A. G. Dias, and R. da Silva, Physica (Amsterdam) 420, 1 (2015).

[43] N. R. F. Braga, M. A. M. Contreras, and S. Diles, Phys. Lett. B 763, 203 (2016).

[44] P. Colangelo, F. De Fazio, F. Giannuzzi, F. Jugeau, and S. Nicotri, Phys. Rev. D 78, 055009 (2008).

[45] S. S. Afonin, Phys. Lett. B 719, 399 (2013).

[46] R. Rougemont, R. Critelli, J. Noronha-Hostler, J. Noronha, and C. Ratti, Phys. Rev. D 96, 014032 (2017).

[47] W. de Paula, T. Frederico, H. Forkel, and M. Beyer, Phys. Rev. D 79, 075019 (2009).

[48] W. de Paula and T. Frederico, Phys. Lett. B 693, 287 (2010).

[49] E. Folco Capossoli, D. Li, and H. Boschi-Filho, Eur. Phys. J. C 76 (2016) 320.

[50] M. Ihl, M. Torres, H. Boschi-Filho, and C. A. B. Bayona, J. High Energy Phys. 09 (2011) 026.

[51] B. Batell, T. Gherghetta, and D. Sword, Phys. Rev. D 78, 116011 (2008).

[52] C. A. Ballon Bayona, H. Boschi-Filho, and N. R. F. Braga, J. High Energy Phys. 03 (2008) 064.

[53] S. Okubo, Phys. Lett. 5, 165 (1963).

[54] F. Xu and M. Huang, Chin. Phys. C 37, 014103 (2013).

[55] A. Cherman, T. D. Cohen, and E. S. Werbos, Phys. Rev. C 79, 045203 (2009).

[56] J. Erlich, E. Katz, D. T. Son, and M. A. Stephanov, Phys. Rev. Lett. 95, 261602 (2005).

[57] S. He, M. Huang, Q. S. Yan, and Y. Yang, Eur. Phys. J. C 66, 187 (2010).

[58] N. R. F. Braga and L. F. Ferreira, Phys. Rev. D 94, 094019 (2016). 